Fr equency and pl asma condi ti on dependent spat i al st ructure of I ow frequency gl obal potential oscillations in the TJ-II st el I ar at or

\begin{tabular}{|l|l|}
\hline $\begin{array}{l}\text { j our nal or } \\
\text { publ i cat i on ti t l e }\end{array}$ & Nucl ear Fusi on \\
\hline vol une & 59 \\
\hline number & 4 \\
\hline page r ange & 044006 \\
\hline year & 2019- 03 05 \\
\hline URL & ht t p: //hdl . handl e. net /10655/00012749 \\
\hline
\end{tabular}




\title{
Frequency and plasma condition dependent spatial structure of low frequency global potential oscilla- tions in TJ-II stellarator
}

\author{
T. Kobayashi ${ }^{1,2}$, U. Losada ${ }^{3}$, B. Liu ${ }^{4}$, T. Estrada ${ }^{3}$, B Ph van Milligen ${ }^{3}$, \\ R. Gerrú ${ }^{3,5}$, M. Sasaki ${ }^{6,7}$, and C. Hidalgo ${ }^{3}$ \\ ${ }^{1}$ National Institute for Fusion Science, National Institutes of Natural Sciences, Toki 509-5292, \\ Japan \\ 2 SOKENDAI (The Graduate University for Advanced Studies), Toki 509-5292, Japan \\ ${ }^{3}$ Laboratorio Nacional de Fusión, CIEMAT, 28040 Madrid, Spain \\ ${ }^{4}$ School of Physical Science and Technology, Southwest Jiaotong University, Chengdu 610031, \\ China \\ ${ }^{5}$ Departamento de Física Teórica, Universidad Autónoma de Madrid, 28049 Madrid, Spain \\ ${ }^{6}$ Research Institute for Applied Mechanics, Kyushu University, Kasuga 816-8580, Japan \\ ${ }^{7}$ Research Center for Plasma Turbulence, Kyushu University, Kasuga 816-8580, Japan \\ E-mail: kobayashi.tatsuya@LHD.nifs.ac.jp
}

\begin{abstract}
In this Letter, we investigate the spatial structure of a low frequency global potential oscillation, which is likely ascribed as the low frequency zonal flows, in TJ-II laboratory plasmas. In two plasmas produced by different heating schemes (electron cyclotron resonance heating and neutral beam injection heating) and characterized by different mean radial electric field structures, frequency-space-decomposed spectra of the global potential oscillation is obtained. In both cases, the oscillatory field has a single-peaked potential structure and a dipole radial electric field structure. The oscillating structure depends on its frequency as well as on the heating scheme.
\end{abstract}




\section{Introduction}

Hierarchy of fields, including global fields and local turbulent fields, exists in a wide variety of systems in nature. For example, nonlinear interaction between global zonal flow and turbulent flow is seen in atmospheric circulation and magnetically confined plasmas, which impacts on dynamics of whole systems. Their generality can be found by the fact that the dynamics are described by the identical equation $[1,2]$. Experiments in laboratory plasmas have a significant advantage that stationary fields can be relatively easily controlled to study the hierarchy of fields. Moreover, importance of the low frequency zonal flows (LFZFs) for turbulent transport regulation in future fusion plasmas has been proposed [3, 4]. A large number of studies including experimental identifications of the LFZFs $[5,6,7,8,9,10,11,12,13]$ as well as theoretical modeling and numerical simulation $[14,15,16,17,18,19,20,21,22,23]$ have been performed to achieve a better understanding of zonal flow nature.

Despite the intensive investigations, spatial structure of the LFZF, which is crucially important to estimate the capability of turbulent transport suppression, is not fully understood. Since the frequency of the LFZF is considered to be zero, direct prediction of the mode structure is difficult, unlike the case of the high frequency branch of the zonal flows [24]. The LFZF is predicted to have a short lifetime, which corresponds to the frequency spectrum broadening [21]. It is also challenging to distinguish the very low frequency component of the LFZF from the equilibrium $E \times B$ flow within such a short duration of the LFZF. Therefore, some statistical approaches having a fine frequency 
resolution are necessary to identify the spatial structure of the LFZF. Although there are some theoretical attempts to predict the $\operatorname{LFZF}$ spatial structure $[15,16,17,18]$, a small number of experimental observations available for examining the theoretical hypotheses hampers further progress. It is claimed that the properties of the LFZFs depend on plasma conditions, e.g., the mean radial electric field $E_{r}$ profile $[9,13]$ or ion mass [12]. In particular, the mean $E_{r}$ profile can modify the turbulent stress that drive the LFZF on the one hand $[25,26,27]$, and the LFZF damping can be altered too by the value of $E_{r}$ on the other hand $[19,20,22]$. However, experimental investigation focusing on the relation between the spatial structure of the LFZFs and the mean $E_{r}$ profile has not been sufficiently performed to validate these theoretical models.

In this Letter, we investigate the spatial structure of a low frequency global potential oscillation, which is likely ascribed as the LFZFs, in the TJ-II stellarator $[9,10,11,12,13]$. The ambipolarity condition has two stable roots in stellarators: the ion root with typically negative $E_{r}$, which is usually achieved in high density plasmas heated by neutral beams, and the electron root with positive $E_{r}$, which is typically realized when electrons are subject to strong heating. The neoclassical transport determines the radial electric field on long (tens of gyroradius) length scales [28, 29]. In two plasmas characterized by the different ambipolarity conditions, frequency-spacedecomposed spectra of the global potential oscillation is obtained. The oscillating $E_{r}$ structure depends on its frequency as well as on the heating scheme. 


\section{Experimental setup}

The TJ-II stellarator is a four-period heliac with the major radius $R=1.5 \mathrm{~m}$ and the averaged minor radius $a=0.2 \mathrm{~m}$. The vacuum magnetic field of $B_{t} \sim 1 \mathrm{~T}$ has a low magnetic shear configuration with the rotational transform at the core $\iota(0) / 2 \pi \sim 1.55$ and that at the plasma surface $\iota(a) / 2 \pi \sim 1.65$ (the standard configuration). The lower safety factor $q=2 \pi / \iota \sim 0.6$ is favorable for driving the LFZFs rather than the geodesic acoustic modes [14]. A favorable MHD stability is realized by a magnetic well configuration.

Floating potential are measured by two systems of Langmuir probe arrays separated poloidally by $\sim 155 \mathrm{deg}$. and toroidally by $\sim 160 \mathrm{deg}$. A radial probe array, the socalled rake probe, consists of 8 tips radially separated by $\sim 1.7 \mathrm{~mm}$ each, whose radial position is scanned on a shot-to-shot basis and is fixed during each discharge. The radial scan covers the range $0.82<\rho<1.02$ with 8 discharges (\#43702-43709), where $\rho$ is normalized averaged minor radius. Another probe, the so-called poloidal probe, which has two tips poloidally separated by $6 \mathrm{~mm}$, is used for evaluating the poloidal structure of fluctuation. Position of the poloidal probe is fixed at $\rho=0.9$ for use as the reference probe for evaluating the long range correlation with respect to the rake probe. 


\section{Results}

\subsection{Target plasmas}

Figure 1 (a) shows a typical time evolution of a target discharge. At the first half of the discharge, the plasma is sustained by two electron cyclotron resonance heating $(\mathrm{ECH})$ systems with the port through power of $250 \mathrm{~kW}$ each, which is deposited at the plasma center. At the middle of the discharge $t=1.15 \mathrm{~s}$, the heating scheme is switched to the co-directed neutral beam injection (NBI) heating having the equivalent port through power to the two ECHs. Time periods of the ECH discharge and the NBI discharge in steady state are $1.08 \leq t \leq 1.13 \mathrm{~s}$ and $1.17 \leq t \leq 1.235 \mathrm{~s}(1.17 \leq t \leq 1.215 \mathrm{~s}$ for \# 43707), respectively, and data in those periods are used for the data analyses below. Both plasmas are in the L-mode state. The ECH plasma has a lower line averaged density and a larger $H_{\alpha}$ emission, which is mainly caused by neutral recycling from the walls induced by outward particle flux, than the NBI plasma. Typical edge plasma parameters are the electron density of $n_{e} \sim 0.3 \times 10^{19} \mathrm{~m}^{-3}$ and the electron temperature of $T_{e} \sim 50 \mathrm{eV}$. Both plasmas have similar collisionality of the order of unity, where the ion temperature $T_{i}$ is assumed to be $T_{i} \sim T_{e}$, and are in the collisional regime (plateau or Pfirsch-Schlüter).

Figure 1 (b) shows the time evolution of the wavelet power spectrum density (PSD) of the floating potential fluctuation, $P(t, f)=X(t, f) X^{*}(t, f)$, where $X(t, f)$ denotes the wavelet decomposition of the signal and $*$ represents the complex conjugate, measured by the reference probe at $\rho=0.9$. Overall, the spectrum is composed by a large 
amplitude oscillation in the low frequency range (defined as $f<20 \mathrm{kHz}$ ) as well as by a turbulent broadband spectrum in the high frequency range $(f>20 \mathrm{kHz})$. Peaks in the low frequency region are split in time and frequency, indicating that the low frequency potential dynamics is characterized by short lifetime sinusoidal-like waveforms, unlike the case reported in [11]. In the ECH period, fluctuation amplitude is larger than that in the NBI period in the entire frequency range.

The radial profiles of the mean floating potential $\bar{V}_{f}$ and its gradient as a proxy of the mean radial electric field $\bar{E}_{r}$ are shown in Figs. 1 (c) and (d), respectively. Data points in those figures correspond to long-time averaged data in each analysis period, and the error bars correspond to the standard error. Curves are the smoothed profile obtained with a numerical lowpass filter, whose cutoff scale is $\Delta \rho \sim 0.033$, because the spatial scale of the neoclassical electric field is expected not to be much finer than that of kinetic profiles. Here, the kerneled moving averaging filter [30] is used as the numerical filter. The error bands of the curves correspond to the absolute value of difference between the data points and the curve, which is smoothed with the same filtering manner afterwards. The finite width of the error bands can be brought by, e.g., imperfect alignment of the probe tips and shot-to-shot difference, which are much larger than the statistical errors of each data points. The $-\nabla \bar{V}_{f}$ profiles presented here are in a qualitative agreement with previously measured $\bar{E}_{r}$ profiles using other diagnostics $[31,32]$. Validity of the use of $-\nabla \bar{V}_{f}$ as a proxy of $\bar{E}_{r}$ is discussed below as well. After the switch of the heating scheme, $-\nabla \bar{V}_{f}$ flips from positive to negative as caused by 
the neoclassical electron-ion root transition. Despite the difference of the polarity, both plasmas have similar $\bar{E}_{r}$ well structures. The plasma in the NBI period has a deeper and narrower well compared to the plasma in the ECH period.

\subsection{Fluctuation spectrum}

Auto PSD of the floating potential fluctuation at $\rho \sim 0.9$ is shown in Figs. 2 (a) and (d) for the ECH discharge and the NBI discharge, respectively. It is defined as $P(f)=\langle P(t, f)\rangle$, where \langle\rangle represents the time average in the time periods for both heating scenarios. Since the turbulence is stationary in the time period of the data analysis, the time average is regarded to be equivalent to the ensemble average. To examine the reproducibility of turbulence spectrum, PSDs from different discharges are overplotted. Since the rake probe is radially scanned between discharges, the PSD from a probe tip closest to $\rho=0.9$ is shown for each discharge. As a result, PSDs in $0.866<\rho<0.927$ are displayed. They appear as almost overlapped curves, showing a reasonable reproducibility of the fluctuation power throughout the discharges. The fluctuation amplitude on the same magnetic surface is approximately equivalent, which is indicated by the mostly overlapping PSDs obtained by different probes. In both heating cases, the shapes of the PSDs are similar. A peak at $f \sim 200 \mathrm{kHz}$ in the NBI period is an energetic ion driven MHD mode.

The radial and poloidal wavenumbers are given as $k_{r, \theta}=\Delta \alpha_{r, \theta} / d_{r, \theta}$, where $d_{r, \theta}$ is the distance of two probe tips measuring the radial and poloidal phase differences 
$\Delta \alpha_{r, \theta}$. The frequency resolved phase difference is given as the complex argument of the cross spectrum density (CSD). The CSD is defined as $C(f)=\left\langle X_{1}(t, f) X_{2}^{*}(t, f)\right\rangle$, where $X_{1}(t, f)$ and $X_{2}(t, f)$ indicate the wavelet spectra of the signals from the pair of the probe tips of the rake probe or the poloidal probe. As is the case above, the pair of the probe tips is chosen to be closest to $\rho=0.9$ in each discharge. The cross phase is only meaningful when it has a nonzero squared cross coherence, i.e., the normalized magnitude of the CSD, defined as $\gamma^{2}(f)=|C(f)|^{2} / P_{1}(f) P_{2}(f)$. The squared cross coherence and the wavenumbers in the radial direction and the poloidal direction are shown in Fig. 2 (b) [(e)] and (c) [(f)], respectively, for the ECH $[\mathrm{NBI}]$ discharge. Results from different discharges are overplotted as in the case of the PSD, showing a reasonable agreement here also. The standard deviation of the phase difference estimate is given as s.d. $\left[\Delta \alpha_{r, \theta}\right]=\left(1-\gamma^{2}\right) /|\gamma| \sqrt{2 n_{\mathrm{d}}}$, where $n_{\mathrm{d}}$ is the number of the ensembles [33] (refer also to [34] for an example of its application). Effective $n_{d}$ depends on the target frequency range because we have employed the wavelet scheme for spectral decomposition. Thanks to the large number of statistically independent ensembles, e.g., more than 1,000 ensembles for $100 \mathrm{kHz}$ fluctuation, uncertainty of cross phase is significantly reduced even with low coherence. Since the standard deviations of the phase difference estimate are much smaller than the shot-to-shot difference, they are not shown here.

As will be discussed below, the long range correlation appears in $f<20 \mathrm{kHz}$, which is in agreement with previous observations $[9,10,12,13]$. In the frequency 
range of $f<20 \mathrm{kHz}$ for the $\mathrm{ECH}$ case and that of $f<10 \mathrm{kHz}$ for the NBI case, $k_{\theta} \sim 0$, which is consistent with a property of the LFZFs. A small but finite $k_{\theta}$ in $10<f<20 \mathrm{kHz}$ in the NBI case can be plausibly explained by linear overlapping of the global LFZF and local turbulences having a finite wavenumber, whose amplitudes are comparable. Looking at the high frequency components in $f>20 \mathrm{kHz}$, signs of both $k_{\theta}$ and $k_{r}$ are opposite in the ECH case and the NBI case, even taking into account a finite scatter of curves. This observation is consistent with the polarity change of $\bar{E}_{r}$. Under the assumption that the turbulence intrinsic phase velocity is negligibly small compared to the background $E \times B$ velocity, the frequency of fluctuations is determined by the $E \times B$ velocity and the poloidal wavenumber as $2 \pi f=k_{\theta} /\left(\bar{E}_{r}^{-1} B_{t}\right)$. Therefore, $k_{\theta, E \times B}=2 \pi f \bar{E}_{r}^{-1} B_{t}$ should meet the measured $k_{\theta}$ if the estimated $E \times B$ velocity from the floating potential measurement is reasonable. Green curves in Fig. 2 (c) and (f) show $k_{\theta, E \times B}$. A reasonable agreement between $k_{\theta}$ and $k_{\theta, E \times B}$ provides a support of use of $-\nabla \bar{V}_{f}$ as a proxy of $\bar{E}_{r}$. A small mismatch between $k_{\theta}$ and $k_{\theta, E \times B}$ may be due to the $\nabla T_{e}$ offset of $\nabla \bar{V}_{f}$ with respect to $\bar{E}_{r}$ and a finite phase velocity of the turbulence.

\subsection{Spatial structure of the low frequency global potential oscillation}

By combining data of the rake probe from different discharges, the spatial structure

of the auto PSD, $P(f, \rho)$, is reconstructed. It is shown in Figs. 3 (a) and (d) for the ECH period and the NBI period, respectively. In the reconstruction, a discontinuity of the spatial pattern that comes from an imperfect reproducibility of the discharges 
is mitigated by normalizing the PSDs with a factor. The factor is defined as $F(f)=$ $P(f, \rho=0.9) / P_{\# 43702}(f, \rho=0.9)$, and shows difference between spectra in discharges and that in the reference discharge (\#43702). Although this factor improves the reconstruction, a small corrugated vertical patterns having a regular separation, which is not real fluctuation structures, still remain. The long range coherence is defined as $\gamma^{2}(f, \rho)=|C(f, \rho)|^{2} / P(f, \rho) P_{\text {ref }}(f)$, where $C(f, \rho)=\left\langle X(t, f, \rho) X_{\text {ref }}^{*}(t, f)\right\rangle$ is the CSD between the tips of the rake probe and the reference probe (a tip of the poloidal probe at $\rho=0.9)$ and $P_{\text {ref }}(f)$ is the power spectrum from the reference probe. It is shown in Figs. 3 (b) and (e). The value of $\gamma^{2}(f, \rho)$ is largest at $\rho=0.9$, where the two probe tips are on the same magnetic surface. In the high frequency range, $f>20 \mathrm{kHz}, \gamma^{2}(f, \rho)$ becomes much smaller, but still nonzero, in both cases. The cross phase with respect to the reference probe, the complex argument of $C(f, \rho)$, is also shown in Figs. 3 (c) and (f). Here, the regions in which the standard deviation of the cross phase estimate is less than $0.1 \times 2 \pi$ are masked. Positive value indicates phase delay with respect to the reference signal. In the low frequency region, $f<20 \mathrm{kHz}$, the phase difference at $\rho \sim 0.9$ is approximately zero in both cases, being in agreement with a feature of the global symmetric oscillation $(m=0$ and $n=0$, where $m$ and $n$ are the poloidal mode number and the toroidal mode number, respectively). The radial variations of the coherence and the phase significantly depend on the frequency. For example, in the ECH plasma, a high value of $\gamma^{2}(f, \rho)$ covers a wider radial range $0.83<\rho<1$, and the phase is almost flat at the lowest frequency range. At higher frequencies the width of the 
structure in $\gamma^{2}(f, \rho)$ is shortened and a phase delay at the edge region becomes finite. On the contrary, in the NBI case, the specific width of the $\gamma^{2}(f, \rho)$ structure increases and the phase delay becomes smaller as the frequency increases up to $f \sim 10 \mathrm{kHz}$.

Structure of the oscillation is further quantified by frequency integration analysis. The frequency integrated PSD and CSD are defined as $\bar{P}(\rho)=\int_{f_{1}}^{f_{2}} P(f, \rho) d f$ and $\bar{C}(\rho)=\int_{f_{1}}^{f_{2}} C(f, \rho) d f$, respectively. By use of them, the frequency integrated squared cross coherence is given as $\bar{\gamma}^{2}(\rho)=|\bar{C}(\rho)|^{2} / \bar{P}(\rho) \bar{P}_{\text {ref }}$. The radial profile of the fluctuation amplitude is given as $\bar{A}(\rho)=\sqrt{\bar{\gamma}^{2}(\rho) \bar{P}(\rho)}$ for a semi-quantitative estimate. The frequency integrated cross phase is given as the complex argument of $\bar{C}(\rho)$ as $\bar{\Theta}(\rho)=\tan ^{-1} \operatorname{Im}[\bar{C}(\rho)] / \operatorname{Re}[\bar{C}(\rho)]$. The frequency width of the integration is taken as $f_{2}-f_{1}=3 \mathrm{kHz}$, in which changes in the oscillation properties are small. Error bars are given according to [33]. Here, the number of independent ensembles that depends on the frequency range is estimated using the frequency center $\left(f_{1}+f_{2}\right) / 2$. Data of $\bar{\Theta}(\rho)$ are shown only in the radial ranges where the error bars are smaller than $0.1 \times 2 \pi$. Radial profiles of $\bar{A}(\rho)$ and $\bar{\Theta}(\rho)$ are shown in the top two rows of Fig. 4 for three different frequency ranges. In both heating cases, the global potential oscillation has a singlepeaked structure within the observable range, whose peak radius depends on the heating scheme. The peak amplitude decreases monotonically as the frequency increases. In the NBI case, a discrete phase inversion exists at $\rho \sim 0.97$. The gradients of $\bar{A}(\rho)$ and $\bar{\Theta}(\rho)$ seem in-out asymmetric with respect to the amplitude peak.

In order to quantify the gradients in $\bar{A}(\rho)$ and $\bar{\Theta}(\rho)$, the radial wavenumber of the 
global potential oscillation is evaluated. Here, the complex wavenumber is introduced as $k_{r}=k_{\mathrm{Re}}+i k_{\mathrm{Im}}$, where $i$ is the imaginary unit. The potential oscillation can be approximated as the local plane wave, $\bar{A}(\rho) \exp [i \bar{\Theta}(\rho)+i \omega t]=\exp \left(i \int k_{\operatorname{Re}} d \rho-\int k_{\operatorname{Im}} d \rho+\right.$ $i \omega t)$, in the both sides of the amplitude peak. Therefore, the real part and the imaginary part of the wavenumber are given as $k_{\mathrm{Re}}=\nabla \bar{\Theta}$ and $k_{\operatorname{Im}}=-\nabla \bar{A} / A$, respectively. The radial gradient is evaluated by the linear fitting within certain radial ranges for both the inner side and the outer side of the amplitude peak, which are shown as the colored rectangles in Fig. 4 top panels. Figures 4 (c) and (f) are the wavenumber of the global potential oscillation as a function of the frequency. In both cases, $k_{\text {Re }}$ and $k_{\operatorname{Im}}$ are highly in-out asymmetric. In the ECH case, an increasing trend of the absolute value of the wavenumbers with respect to the frequency is similar in the inner part and the outer part. However, the magnitude of the wavenumbers from the outer part is much larger than that from the inner part. On the contrary, the case of the NBI is more complicated. The absolute values of the wavenumbers from the inner part have a decreasing trend with the frequency. For the outer part, the imaginary part of the wavenumber dominates the real part of the wavenumber. The value of the imaginary part of the wavenumber weakly depends on the frequency.

To examine robustness of the discussion above, another attempt of the gradient evaluation is performed with $80 \%$ of the radial range (10\% each from both sides). The result obtained remains qualitatively unchanged. The frequency dependence of the global potential oscillation wavenumber is shown for the first time. In addition, different 
features are observed depending on the plasma operation condition. The collisionarity dependence of the zero frequency zonal flow wavenumber has been quantified in JET [8]. As is stated above, the collisionarities of both discharges are in the same regime so that the difference in oscillation characteristics seems to be ascribed to the difference in polarity and structure of the global radial electric field.

By use of the amplitude and phase of the global potential oscillation, the waveform of the radial electric field oscillation is reconstructed as $E_{r}=-\nabla[A \cos (-f \tau+\Theta)]$. Before the reconstruction, smoothing by use of a numerical lowpass filter is performed for $A$ and $\Theta$ to remove shot-to-shot scatter of the points. Figures 5 (a) and (c) show the reconstructed waveforms as a function of the frequency and the radius for the instance of $f \tau=0$. Here, the band width of the frequency integration is $3 \mathrm{kHz}$ as the case of Fig. 3, but data at up to the higher frequency are shown. In the NBI case, a clear dipole structure of the oscillatory $E_{r}$ can be seen. The amplitude is large only when the frequency is low. In the ECH case, the radial structure of the oscillatory $E_{r}$ seems to be wider. Although the range $\rho<0.82$ is out of observable radius, from a decreasing trend in the $V_{f}$ amplitude [Fig. 4 (a)], the counterpart of the dipole $E_{r}$ structure is expected to exist, and to compose the dipole structure as well. In contrast to the NBI case, the frequency dependence of the $E_{r}$ amplitude is weak. From the series of analyses, it is confirmed that a preferential oscillatory state, in terms of the frequency and the spatial structure, exists depending on plasma conditions. Phase inversion point of the dipole structure at the lowest frequency range seems to approximately agree to the inner shear 
region in both cases. An interpretation, the lowest frequency structure is smoothly connected to the zero frequency structure, is possible.

The bottom plots are snapshots of the $E_{r}$ oscillations at $f \tau=0$ and $\pi$, overlapped on top of the mean $E_{r}$ profiles. Although the values of $E_{r}$ profiles have relatively large errors as shown Fig. 1 (d), we omit them. Therefore, the snapshots show representative examples of how they evolve in space and time. These figures correspond to possible illustrations of the $E_{r}$ behavior. As the mean $E_{r}$ profiles have relatively large errors as shown Fig. 1 (d), the actual mean $E_{r}$ values can have some offsets, which are not so much interested in. By the low frequency global $E_{r}$ oscillation, the well depth of the $E_{r}$ profile is modulated in both cases. Therefore, the $E_{r}$ shear and the $E_{r}$ curvature are modulated by the oscillation, which can impact the transport regulation as discussed in $[10,11]$

\section{Summary}

In this Letter, we showed frequency and plasma condition dependent structures of the global radial electric field oscillation. Results reported here show, with unprecedented detail, how sensitive are the properties of zonal flows to plasma conditions. These provide a key experimental guide for model validation. Whether this sensitivity is due to the magnitude or sign of radial neoclassical electric fields affecting Reynolds stress driven zonal flows and neoclassical mechanisms [20, 35] or/and plasma profile effects remain an open question. We also note that the external parallel momentum input 
should also impacts on the zonal flow dynamics. These experimental details will be assessed in the future investigations.

Another important topic on the zonal flows is the isotope mass dependence $[22,23,12,36]$. Theoretical studies claim that the isotope mass dependence appears in a specific spatial scale regime [23]. Therefore, the approach used in this paper, i.e., observation of spatial structures with a frequency resolution, would be beneficial to reveal that theoretical proposal. Further experimental activities focusing on the isotope mass dependence are planned in future.

\section{Acknowledgments}

One of the authors (TK) acknowledges Dr. K. Ida, Dr. M. Yokoyama, Dr. N. Tamura, and Dr. S. Sakakibara for strong support. This work is supported by NINS (National Institutes of Natural Sciences)/NIFS (National Institute for Fusion Science) under the project, "Strategic International Research Interaction Acceleration Initiative" and by the Grant-in-Aid for Scientific Research of Japan Society for the Promotion of Science (17K14898). 


\section{References}

[1] J G Charney 1948 Geophys. Publ. 171

[2] A Hasegawa and K Mima 1978 Phys. Fluids 21 87-92

[3] Z Lin et al 1998 Science $\mathbf{2 8 1} 1835$

[4] PH Diamond, SI Itoh, K Itoh, and TS Hahm 2005 Plasma Phys. Control. Fusion 47 R35

[5] A Fujisawa et al 2004 Phys. Rev. Lett. 93165002

[6] DK Gupta et al 2006 Phys. Rev. Lett. 97125002

[7] AD Liu et al 2009 Phys. Rev. Lett. 103095002

[8] JC Hillesheim et al 2016 Phys. Rev. Lett. 116065002

[9] MA Pedrosa et al 2008 Phys. Rev. Lett. 100215003

[10] C Silva et al 2011 Nucl. Fusion 51063025

[11] JA Alonso et al 2012 Nucl. Fusion 52063010

[12] B Liu et al 2015 Nucl. Fusion 55112002

[13] U Losada et al 2016 Plasma Phys. Control. Fusion 58084005

[14] N Miyato et al 2004 Phys. Plasmas 11 5557-5564

[15] L Chen et al 2000 Phys. Plasmas 7 3129-3132

[16] AI Smolyakov et al 2000 Phys. Rev. Lett. 84491

[17] P Kaw et al 2001 Plasma Phys. Control. Fusion 4451

[18] K Itoh et al 2004 J. Phys. Soc. Jpn. 73 2921-2923

[19] K Itoh et al 2007 Phys. Plasmas 14020702

[20] JL Velasco et al 2012 Phys. Rev. Lett. 109135003

[21] TS Hahm, KH Burrell, Z Lin, R Nazikian, and EJ Synakowski 2000 Plasma Phys. Control. Fusion 42 A205

[22] TH Watanabe, H Sugama, and M Nunami 2011 Nucl. Fusion 51123003

[23] TS Hahm, Lu Wang, WX Wang, ES Yoon, and FX Duthoit 2013 Nucl. Fusion 53072002 
[24] K Itoh, S-I Itoh, P H Diamond, A Fujisawa, M Yagi, T Watari, et al 2006 Plasma Fusion Res. 1 $037-037$

[25] H Biglari, PH Diamond, and PW Terry 1990 Phys. Fluids B 2 1-4

[26] E-J Kim and PH Diamond 2003 Phys. Plasmas 10 1698-1704

[27] M Sasaki et al 2018 Phys. Plasmas 25012316

[28] P Helander and AN Simakov 2008 Phys. Rev. Lett. 101145003

[29] I Calvo et al 2013 Plasma Phys. Control. Fusion 55125014

[30] P Bloomfield. Fourier analysis of time series (Chapter 7). Wiley. com, 2004.

[31] T Estrada et al 2009 Plasma Phys. Control. Fusion 51124015

[32] AV Melnikov et al 2011 Nucl. Fusion 51083043

[33] JS Bendat and AG Piersol. Random data (Chapter 9). John Wiley \& Sons, New York, 2000.

[34] A E White, W A Peebles, T L Rhodes, C H Holland, G Wang, L Schmitz, T A Carter, J C Hillesheim, E J Doyle, L Zeng, et al 2010 Phys. Plasmas 17056103

[35] J Guasp and M Liniers 2000 Nucl. Fusion 40411

[36] Y Xu, C Hidalgo, I Shesterikov, A Krämer-Flecken, S Zoletnik, M Van Schoor, M Vergote, Textor Team, et al 2013 Phys. Rev. Lett. 110265005 

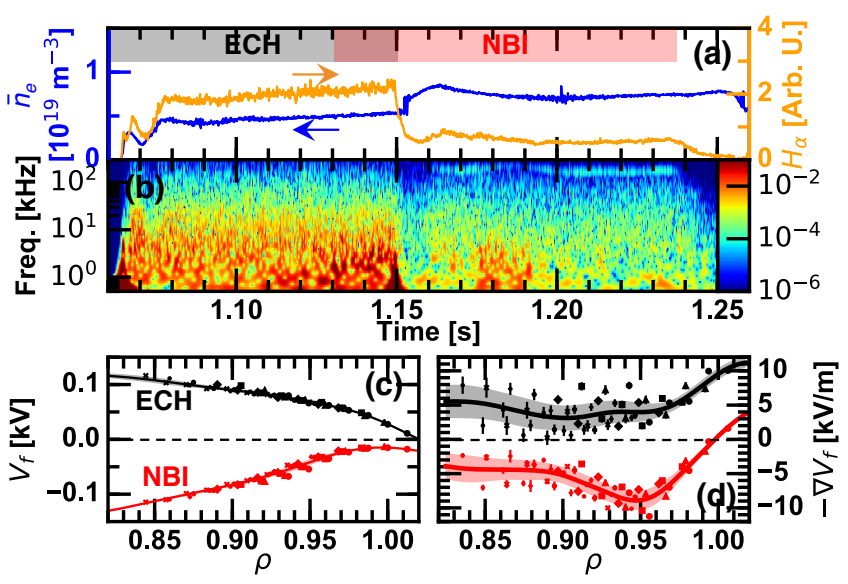

Figure 1. Time evolutions of (a) the line averaged density $\bar{n}_{e}$ and the $H_{\alpha}$ emission intensity,

(b) wavelet power spectral density of floating potential at $\rho \sim 0.9$, radial profiles of (c) mean floating potential and (d) mean radial electric field averaged in the ECH period and the NBI period. Different symbols in (c) and (d) show the long-time averaged data from different shots.
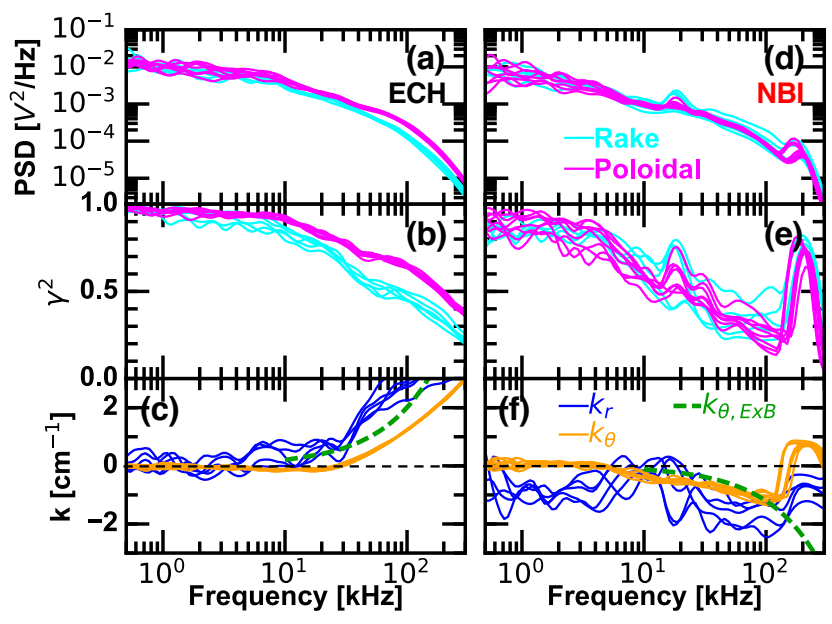

Figure 2. Left: (a) Auto power spectral density of the floating potential fluctuation at $\rho \sim 0.9$,

(b) squared cross coherence between probe pairs, and (c) frequency-resolved radial wavenumber and poloidal wavenumber in the ECH period. Right: (d-f) Those for NBI period. 


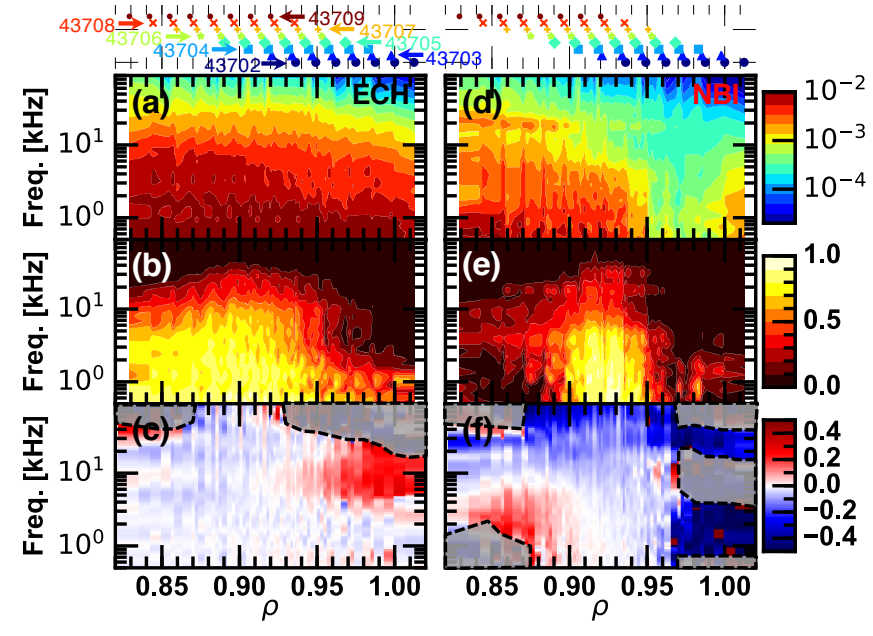

Figure 3. Left: Frequency-space-resolved (a) power spectral density of the floating potential fluctuation and (b, c) long range cross coherence and cross phase in the ECH period. Right: (d-f) Those in the NBI period. Correspondence between shot numbers and the radial position of the rake probe are shown by symbols at the top of figures.

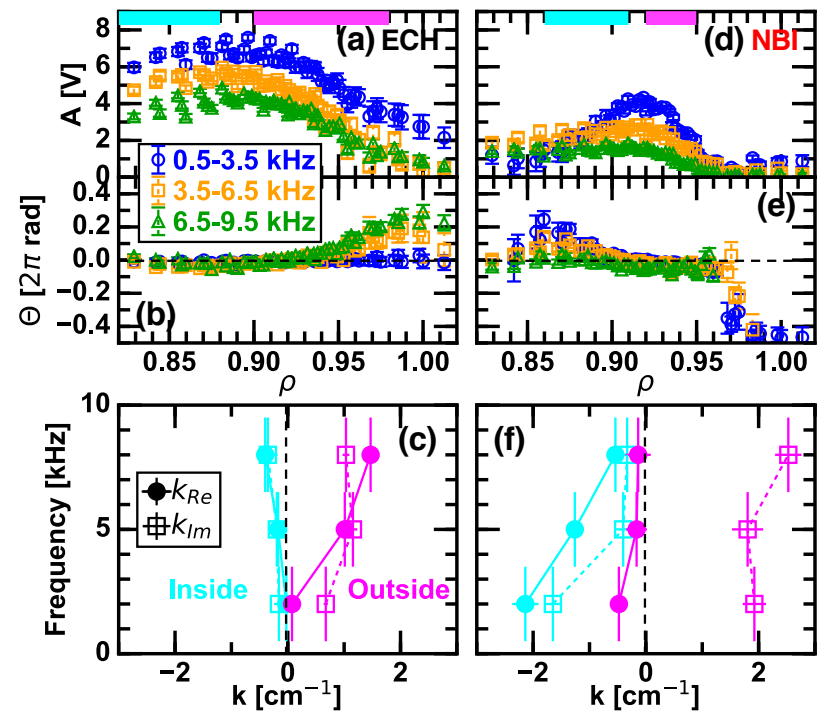

Figure 4. Left: Frequency-integrated (a) fluctuation amplitude and (b) phase for three different frequency ranges, and (c) radial wavenumber of the low frequency global potential oscillation fitted at the inner side (cyan) and the outer side (magenta) of the amplitude peak in the ECH discharges. The regions for the fitting indicated by the horizontal thick lines in top panels. Right: (d-f) Those in the NBI discharges. 


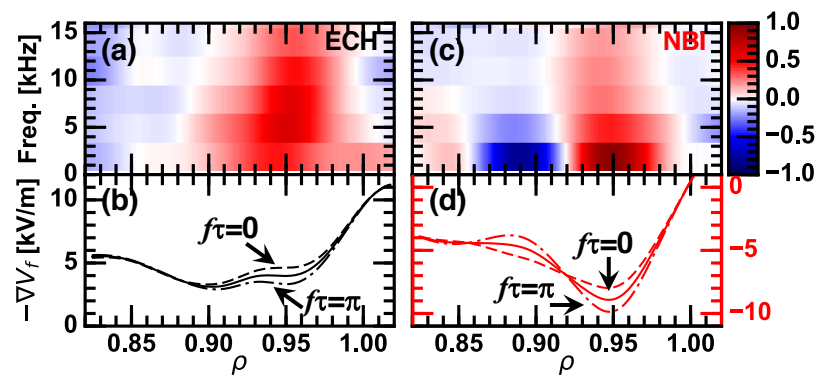

Figure 5. Left: Reconstructed waveforms of the low frequency global oscillation in radial electric field (a) plotted as a function of the frequency and (b) plotted on top of the mean profile in the ECH discharges. Right: (c) and (d) Those in the NBI discharges. Frequency ranges used for (b) and (d) are $3.5-6.5 \mathrm{kHz}$ and $0.5-3.5 \mathrm{kHz}$, respectively. 